Consent concern:

Forms bogged down in legal jargon fail to keep people informed

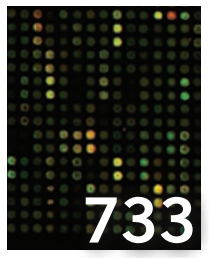

Data depot:

Researchers aim to protect their data in lasting repositories

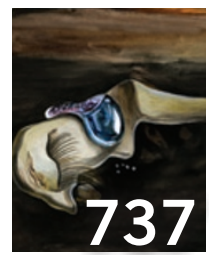

Long shot:

Injectables that last

for weeks help treat

psychiatric disorders

\title{
Lack of microarray uptake compromises diagnosis of young
}

For more than three decades, the first-line test for spotting genetic disorders in young children has been a basic laboratory assay in which a technician analyzes a toddler's chromosomes under the microscope for unusual structural rearrangements. About four years ago, a new technology based on fluorescent probes hit the scene and, in short order, became the default assay for most testing labs.

But some insurance companies have resisted paying for the newer tests, called chromosomal microarrays, because they are more expensive than older techniques. This delay in technological uptake could be keeping many children from receiving crucial early treatment for their conditions. Now, an expert group is calling on large medical associations to adopt microarrays as the preferred genetic tests for children with unexplained autism, developmental delays or other birth defects.

Traditional karyotyping techniques are still the best choice for conditions such as Down's syndrome that are caused by gross chromosomal abnormalities and are easily recognized by clinicians. But most developmental disorders show a range of symptoms and can arise from more subtle genetic glitches, such as microscopic DNA deletions or duplications. That's where the much more sensitive microarrays come in.

Microarrays work by breaking down samples of DNA into tiny bits and then comparing them for their ability to hybridize with a library of known targets. The first highthroughput microarrays used complementary DNA (converted from messenger RNA) as a measure of gene expression. Newer types, in contrast, recognize the DNA itself. For instance, array-based comparative genomic hybridization allows for higher-resolution detection of structural variants including DNA deletions and duplications. Single nucleotide polymorphism arrays, a similar innovation, pick up single-letter blips in the genetic code to test for associations of common genetic variants to a wide range of human diseases.

In a meta-analysis of 33 studies, clinical

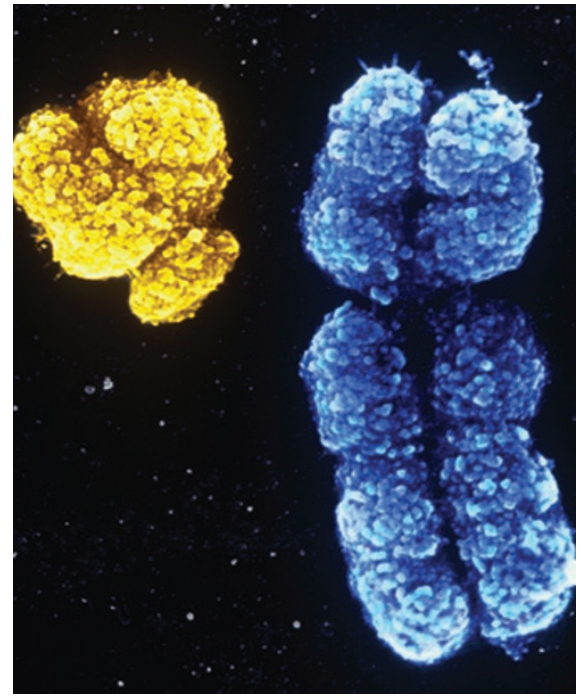

Karyotype gripe: More microarrays needed.

geneticists from the International Standard Cytogenomic Array Consortium, a group formed in 2008 with the goal of standardizing microarray methods, reported that chromosomal microarray tests identified the genetic culprit in an average of $12 \%$ of children with various developmental disabilities with previously unknown genetic and nongenetic causes, compared to a hit rate of only around $2 \%$ for karyotype staining. The findings appeared in the May issue of the American Journal of Human Genetics (86, 749-764, 2010).

Pinpointing a known genetic syndrome can directly improve medical care, says study author David Miller, a clinical geneticist at Children's Hospital Boston. For instance, a toddler with developmental delay may carry a deletion known to also cause heart defects. "If you found that, then you would consider having them go see the cardiologist," he says.

\section{Hefty price tag}

Miller estimates that his Boston lab performs around 300 microarray tests each month. But the same isn't true of smaller community clinics, which don't have such deep pockets. To offer in-house testing, a clinic would need to spend at least $\$ 200,000$ on new equipment, plus the expense of technician training. And without the facilities, many physicians have a tough time convincing insurance companies to pay for microarray tests from commercial labs, which, at around $\$ 1,500-\$ 2,000$ apiece, cost more than twice the amount karyotyping does.

Still, some argue that microarray testing makes financial sense. In the same issue of the American Journal of Human Genetics, health economists in the UK and Canada used mathematical models to compare the two strategies. They found that, for every 100 children tested, microarray testing made around eight more causal diagnoses than karyotyping did, and this was at an extra cost of only around $\$ 200$ per patient ( $\mathrm{Am}$. J. Hum. Genet. 86, 765-772, 2010). Other experts point out that the additional expense may pay for itself, because physicians often order pricey additional tests, such as brain imaging scans, when karyotyping does not produce clear results.

There may be other, less obvious savings, too, notes Gurdeep Sagoo, an epidemiologist at the PHG Foundation in Cambridge, UK who studies the financial and public health implications of genetic testing. For example, microarray testing takes about half the time of karyotyping, and the machines can be used for other applications, such as cancer diagnoses, he says.

Microarray tests can be tricky to interpret, however, because they often detect genetic variations that have not yet been linked to disease. "It became obvious very early that any one lab would not have sufficient data to gain the empiric evidence of what was clinically significant versus benign," says David Ledbetter, director of medical genetics at Emory University in Atlanta and head of the International Standard Cytogenomic Array Consortium. To help fill in the gaps, the group launched a database in May to pool genetic data from researchers across the US. The continuously growing resource now holds microarray data from nearly 16,000 individuals.

Virginia Hughes, New York 